\title{
The Application of Invariant Imbedding to the Solution of Linear Two-Point Boundary Value Problems on an Infinite Interval
}

\author{
By Dale W. Alspaugh
}

\begin{abstract}
Linear two-point boundary value problems defined on an infinite domain are converted to initial-value problems using invariant imbedding. The resulting Riccati equations are integrated numerically until the desired accuracy is obtained. Several criteria for determining the appropriate length of integration are presented. Several example problems are presented.
\end{abstract}

1. Introduction. Many problems of practical significance are conveniently modeled by assuming that the domain of the governing equations is infinite or semi-infinite. When analytic solutions can be obtained, this assumption often simplifies the task of solution and the form in which the solutions are expressed. As is often the case however, analytic solutions are not readily attainable or may not exist in a form expressible by elementary functions. In such cases, the analyst is brought to the problem of devising a feasible algorithm for numerical solution of the governing equations. The simplification that accrued to the analytic solution by virtue of the formulation as a problem on an infinite domain is now replaced by the difficulty of dealing with an infinite domain by means of a finite machine. The usual response to this type of problem is to assume that the domain is large but finite. Immediately, one must attend to the decision as to how large the domain must be in order to properly represent an infinite domain in the computational sense.

In a recent paper, Robertson [1] presented a finite-difference scheme for treating linear two point boundary value problems (TPBVP) on an infinite interval. In this paper, we present two variations on the use of invariant imbedding to treat the linear TPBVP on an infinite interval. Invariant imbedding is a technique that converts a TPBVP to an initial-value problem. Obviously, initial-value problems are well suited to numerical solution by the use of a digital computer. Several recent papers [2], [3] , [4] and [5] present the invariant imbedding formulation and solution of a variety of

Received August 16, 1973.

AMS (MOS) subject classifications (1970). Primary 65L10, 68 L04; Secondary 34B05.

Key words and phrases. Boundary value problems, invariant imbedding, infinite domain. 
problems. These papers discuss, in some detail, the formulation and validate the invariant imbedding solutions. Two new books [6], [7] on invariant imbedding also treat a number of example applications. In this paper, we shall outline the formulation and proceed to the solution.

2. Problem Definition. In this paper, we restrict our attention to the solution of linear two-point boundary value problems governed by a second-order linear differential equation of the form

$$
L\{y(x)\} \equiv y^{\prime \prime}+p(x) y^{\prime}+q(x) y=f(x)
$$

and the boundary conditions

$$
y(a)=y_{0}, \quad \lim _{L \rightarrow \infty} y(L)=y_{f} .
$$

3. Invariant Imbedding Formulation. As was mentioned previously, earlier works have dealt extensively with the methods of formulation and validation of the invariant imbedding method as applied to various types of problems. In this paper, we shall briefly sketch the formulation using invariant imbedding. We presume, however, no previous knowledge of invariant imbedding.

The basis of the method to be described is the imbedding of the problem of interest in a family of problems of wider scope and solving this larger class of problems. At first, this seems more difficult than the original problem; however, as we shall show, a computationally simple problem results from this imbedding. For the class of problems under discussion, the imbedding parameter is the length of the interval over which the system of equations is solved.

In the problem at hand, we shall take advantage of the linearity of the governing differential equations and boundary conditions. Nonlinear problems can also be treated, however, certain added complications arise and will be treated in subsequent work. We first assume that the dependent variable $y(x)$ can be written as

$$
y(x)=u(x, L)+y_{0} v(x, L)+y_{f} w(x, L) .
$$

Note that the new dependent variables are shown as explicit functions of both $x$ and the length of the interval $L$. On substitution of Eq. (3) into (1) and (2), we choose the dependent variables so as to satisfy the following equations and boundary conditions:

$$
\begin{aligned}
L\{u\} & =f(x), & L\{v\} & =0, & L\{w\} & =0, \\
u(a, L) & =0, & v(a, L) & =1, & w(a, L) & =0, \\
u(L, L) & =0, & v(L, L) & =0, & w(L, L) & =1 .
\end{aligned}
$$

It is clear that the superposition of the solution of Eq. (4) will satisfy Eqs. (1) and (2) when $L \rightarrow \infty$. 
In the work that follows, a prime will be used to denote the derivative of a function with respect to its first argument and a subscript 2 to denote the derivative with respect to the second argument. We begin the derivation by differentiating the differential equations and boundary conditions of Eq. (4) with respect to the length of the interval $L$. Since the linear operator $L$ does not involve the interval length $L$, the differentiation may be carried inside the operator symbol and we obtain

$$
\begin{aligned}
& L\left\{u_{2}(x, L)\right\}=0, \quad L\left\{v_{2}(x, L)\right\}=0, \quad L\left\{w_{2}(x, L)\right\}=0, \\
& u_{2}(a, L)=0, \quad v_{2}(a, L)=0, \quad w_{L}(a, L)=0, \\
& u^{\prime}(L, L)+u_{2}(L, L)=0, \quad v^{\prime}(L, L)+v_{2}(L, L)=0, \quad w^{\prime}(L, L)+w_{2}(L, L)=0 .
\end{aligned}
$$

Note that the variables $u_{2}, v_{2}, w_{2}$ and $w$ all satisfy the same linear differential equation and boundary conditions of the same general form. Thus, we may write

$$
\begin{gathered}
u_{2}(x, L)=-u^{\prime}(L, L) w(x, L), \\
v_{2}(x, L)=-v^{\prime}(L, L) w(x, L), \\
w_{2}(x, L)=-w^{\prime}(L, L) w(x, L) .
\end{gathered}
$$

Equations (6) are differential equations in the independent variable $L$ with $x$ as a parameter. If $u^{\prime}, v^{\prime}$ and $w^{\prime}$ are known functions of $L$, the system of Eq. (6) can be integrated. The initial conditions for such an integration will be shown in a subsequent paragraph.

At this point, we define three functions of the interval length $L$ as

$$
r(L)=u^{\prime}(L, L), \quad s(L)=v^{\prime}(L, L), \quad t(L)=w^{\prime}(L, L) .
$$

Differentiating the first of Eq. (7) with respect to $L$ gives

$$
r^{\prime}(L)=u^{\prime \prime}(L, L)+u_{2}^{\prime}(L, L) .
$$

But from Eqs. (1) and (4),

$$
u^{\prime \prime}(L, L)=-p(L) u^{\prime}(L, L)-q(L) u(L, L)+f(L) .
$$

Applying the boundary conditions of Eq. (4) and the definition in Eq. (7), we find

$$
u^{\prime \prime}(L, L)=-p(L) r(L)+f(L) .
$$

From the first of Eq. (6) $u_{2}^{\prime}(x, L)=-u^{\prime}(L, L) w^{\prime}(x, L)$ or, when $x=L$,

$$
u_{2}^{\prime}(L, L)=-r(L) t(L) .
$$

On substituting Eqs. (10) and (11) in (8), we find

$$
r^{\prime}(L)=-p(L) r(L)+f(L)-r(L) t(L) .
$$


In a similar fashion, differential equations for $s$ and $t$ are derived. The complete set of equations is summarized as follows:

$$
r^{\prime}=-(p+t) r+f(L), \quad s^{\prime}=-(p+t) s, \quad t^{\prime}=-(p+t) t-q(L) .
$$

The initial conditions associated with the system of differential equations are found by noting from Eq. (4) that $\lim _{L \rightarrow a} u(a, L)=u(a, a)$ which implies that $u^{\prime}(a, a) \equiv$ $r(a)=0$. In a similar fashion the following set of initial conditions is obtained

$$
r(a)=0, \quad s(a)=-\infty, \quad t(a)=\infty .
$$

Using the boundary conditions of Eq. (4), it is easily shown that the following conditions are also satisfied

$$
u(x, x)=0, \quad v(x, x)=0, \quad w(x, x)=1 .
$$

It is of interest to note that, if the functions $p(L), q(L)$ and $f(L)$ are assumed to asymptotically approach the values $p_{\infty}, q_{\infty}$ and $f_{\infty}$ as $L$ approaches infinity, the system of equations (13) has the asymptotic solution

$$
t_{\infty}=\frac{-p_{\infty} \pm \sqrt{p_{\infty}^{2}-4 q_{\infty}}}{2}, \quad s_{\infty}=0, \quad r_{\infty}=\frac{2 f_{\infty}}{p_{\infty} \pm \sqrt{p_{\infty}^{2}-4 q_{\infty}}}
$$

4. Invariant Imbedding Solution. In order to obtain the solution of the twopoint boundary value problem, the system of Riccati equations (13) is integrated numerically using the initial conditions prescribed in Eq. (14). Any standard numerical ${ }^{\circ}$ integration scheme can be used. Experience has shown that the equations are numerically stable.* Note that Eq. (14) are differential equations for the magnitudes of unknown terminal conditions; the independent variable is the interval length.

The integration is continued until the interval adequately approximates the semiinfinite domain. In a later section, we discuss means for choosing the interval length to terminate the integration. Note that, when the integration has proceeded to an acceptable magnitude, the values of $r, s$, and $t$ at that point could be combined to give the unknown value of the slope at "infinity" through the relationship

$$
y^{\prime}\left(L_{\infty}\right)=r\left(L_{\infty}\right)+y_{0} s\left(L_{\infty}\right)+y_{\infty} t\left(L_{\infty}\right) \text {. }
$$

The system equations, Eq. (1), could then be integrated backwards from $L_{\infty}$ to $a$ and the solution to the TPBVP thus obtained. While this method is correct, numerical difficulties may be encountered in cases where the solution is highly sensitive to the initial conditions as, for example, when the roots of the characteristic equation are widely separated.

*The term "numerical stability" is used in the sense that errors made in one stage of the computation are not propagated into larger errors in later stages of the computation. 
In order to circumvent these numerical difficulties, it is the usual practice to append a set of equations of the form

$$
\begin{aligned}
u_{2}(x, L) & =-r(L) w(x, L), \\
v_{2}(x, L) & =-s(L) w(x, L), \quad L \geqslant x, \\
w_{2}(x, L) & =-t(L) w(x, L),
\end{aligned}
$$

to Eq. (13) for each value of $x$ for which output is desired. Equations (18) are then integrated simultaneously with (13) using the conditions given in Eq. (15). Experience has shown that this system of equations is also numerically stable. The solution to the TPBVP is then the superposition given in Eq. (3).

The numerical difficulties imposed by the infinite initial conditions can easily be circumvented using a simple transformation suggested by Wing [5] and used previously by the author in [2], [3]. To begin the integration of Eq. (13) the following transformations are introduced

$$
S=1 / s, \quad T=1 / t .
$$

The differential equations (13) are then transformed according to Eq. (19) and L'Hospital's rule applied to determine the derivatives at the initial point. The transformed equations are integrated for a short distance until the transformed variables become nonzero. At this point, transformation is made back to the original system and the integration continued. The same transformation is made any time that any of the variables begin to grow rapidly. This process of inversion allows smooth passage through the points at which infinite solutions are obtained.

One of the central problems in the numerical solution of two-point boundary value problems defined on an infinite domain is the determination of an interval length that will result in an acceptable approximation to infinity. Standard methods require some a priori estimation of the proper interval length. If by some means it is found that the chosen length was inappropriate, it is necessary to repeat the entire process with a new interval.

Invariant imbedding requires no a priori estimate of the appropriate interval. Integration is simply continued until some measure of the accuracy of the solution indicates that satisfactory results have been achieved. Several possible criteria exist that can be utilized. For example, the solution at one or more points can be observed and the integration terminated when apparent "convergence" of the solution is obtained. This method can be used to advantage when a particular range of the independent variable is of primary interest.

One criterion investigated in the work presented in this paper is based on analytic computation of the rate of change of the solution with respect to the interval length. On differentiating Eq. (3) with respect to $L$ and utilizing Eqs. (6) and (7), 


$$
d y / d L=-\left(r+y_{0} s+y_{f} t\right) w(x, L) .
$$

From Eq. (20) it is seen that the greatest effect of the interval length change is associated with the maximum value of $w(x, L)$ and that a sufficient condition for the existence of an asymptotic solution is that the quantity

$$
E(L) \equiv\left|r(L)+y_{0} s(L)+y_{f} t(t)\right|
$$

approach zero. $E$ can thus serve as a convergence indicator.

Another related criterion was obtained by computing the square of the average rate of change over the entire interval. Let

$$
E^{2}(L) \equiv \frac{1}{L^{2}} \int_{0}^{L}\left(\frac{d y}{d L}\right)^{2} d x .
$$

From Eq. (20),

$$
E^{2}(L)=\frac{1}{L^{2}} \int_{0}^{L}\left(r+y_{0} s+y_{f} t\right)^{2} w^{2}(x, L) d x
$$

or

$$
E^{2}(L)=\frac{\left(r+y_{0} s+y_{f} t\right)^{2}}{L^{2}} \int_{0}^{L} w^{2}(x, L) d x
$$

Let $I(L)=\int_{0}^{L} w^{2}(x, L) d x$; then

$$
\frac{d I}{d L}=w^{2}(L, L)+\int_{0}^{L} w w_{2} d x
$$

But from Eqs. (6) and (7), $w_{2}(x, L)=-t(L) w(x, L)$, so

$$
\frac{d I}{d L}=1-t \int_{0}^{L} w^{2}(x, L) d x
$$

or

$$
\frac{d I}{d L}+t(L) I(L)=1
$$

Note that, if $t$ approaches a limit $\left(t_{\infty}\right)$ asymptotically,

$$
\lim _{L \rightarrow \infty} I(L)=1 / t_{\infty}
$$

Thus, under weak restrictions on the asymptotic behavior of $r, s$ and $t$,

$$
\lim _{L \rightarrow \infty} E(L)=0
$$


A stopping condition based on this criterion can be obtained by continuing the integration until the quantity

$$
E(L) \equiv\left|\left(r+y_{0} s+y_{f} t\right) \sqrt{I(L)}\right| / L
$$

is reduced to some specified value.

Under certain circumstances, an alternative means of superposing the individual solutions may be used to provide a somewhat faster convergence. If no conditions, other than finiteness, are to be imposed at infinity, or if $y_{f}$ equals zero, the following alternate approach may be used. Note that the slope of the solution at the end of the interval is given by

$$
\left.\frac{d y}{d x}\right|_{L}=u^{\prime}(L, L)+y_{0} v^{\prime}(L, L)+y_{f} w^{\prime}(L, L)
$$

or

$$
\left.\frac{d y}{d x}\right|_{L}=r+y_{0} s+y_{f} t
$$

Assuming that $y_{f}=0$, the slope at infinity is given by

$$
\left.\lim _{L \rightarrow \infty} \frac{d y}{d x}\right|_{L}=r_{\infty}+y_{0} s_{\infty}
$$

This suggests the alternate superposition of the solutions $u, v$ and $w$

$$
y(x, L)=u(x, L)+y_{0} v(x, L)+C u(x, L)
$$

where

$$
C=\left[r_{\infty}-r(L)\right]+y_{0}\left[s_{\infty}-s(L)\right] / t(L)
$$

5. Example Problems. In order to illustrate the technique, several example problems have been solved and are presented in this section. The first example was solved by Robertson [1]. The governing equation is

$$
d^{2} y / d x^{2}-(1+1 / x) y=-1 / x^{2}
$$

subject to the boundary conditions $y(1)=0 \lim _{L \rightarrow \infty} y(L)=0$. As Robertson points out, the solution of this problem approaches the asymptotic solution relatively slowly. The results presented in this paper were obtained by means of a FORTRAN program run on a CDC 6500 digital computer. However, a nearly identical program was successfully run on a much smaller computer (IBM 1130). The CDC machine was used simply because it was desired to produce the curves for this paper on a Calcomp plotter.

Fig. 1 shows the results of the application of the Invariant Imbedding method to this problem. The various curves show the distribution of $y(x)$ for the various 


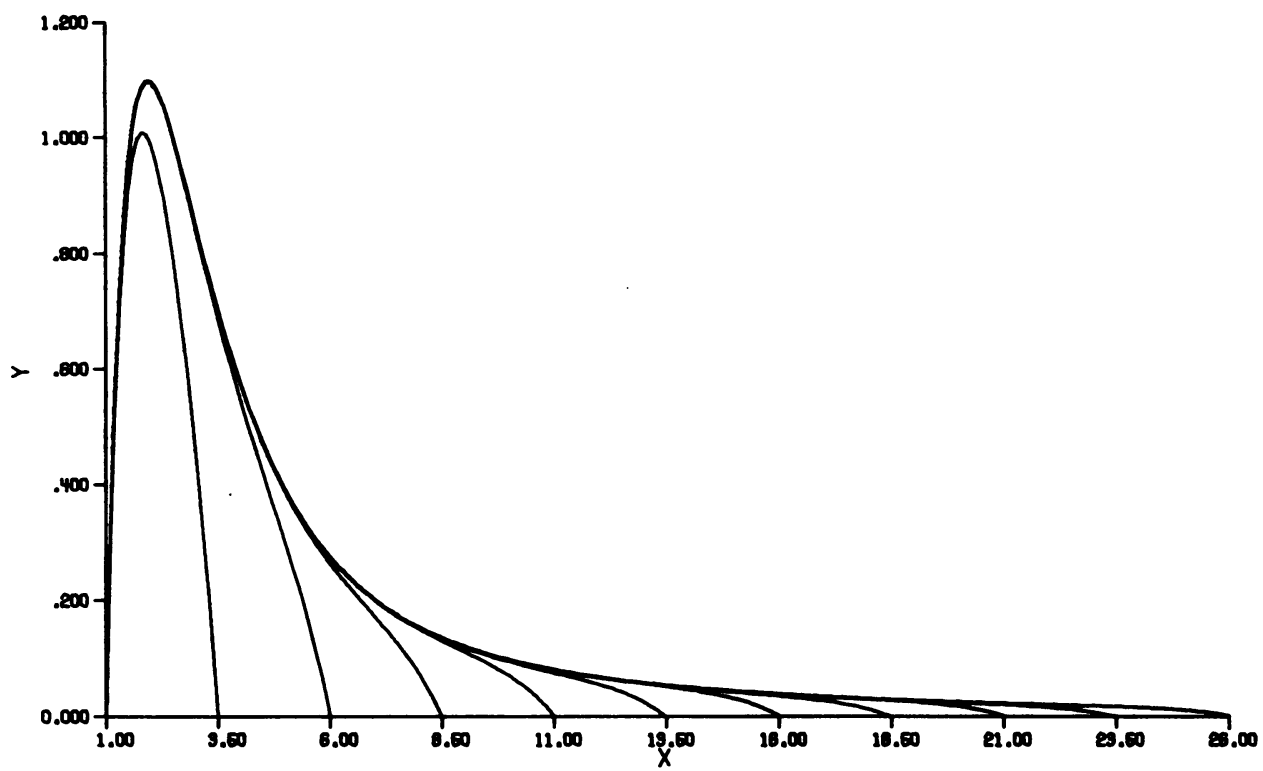

FIGURE 1. Invariant Imbedding Solution, First Example

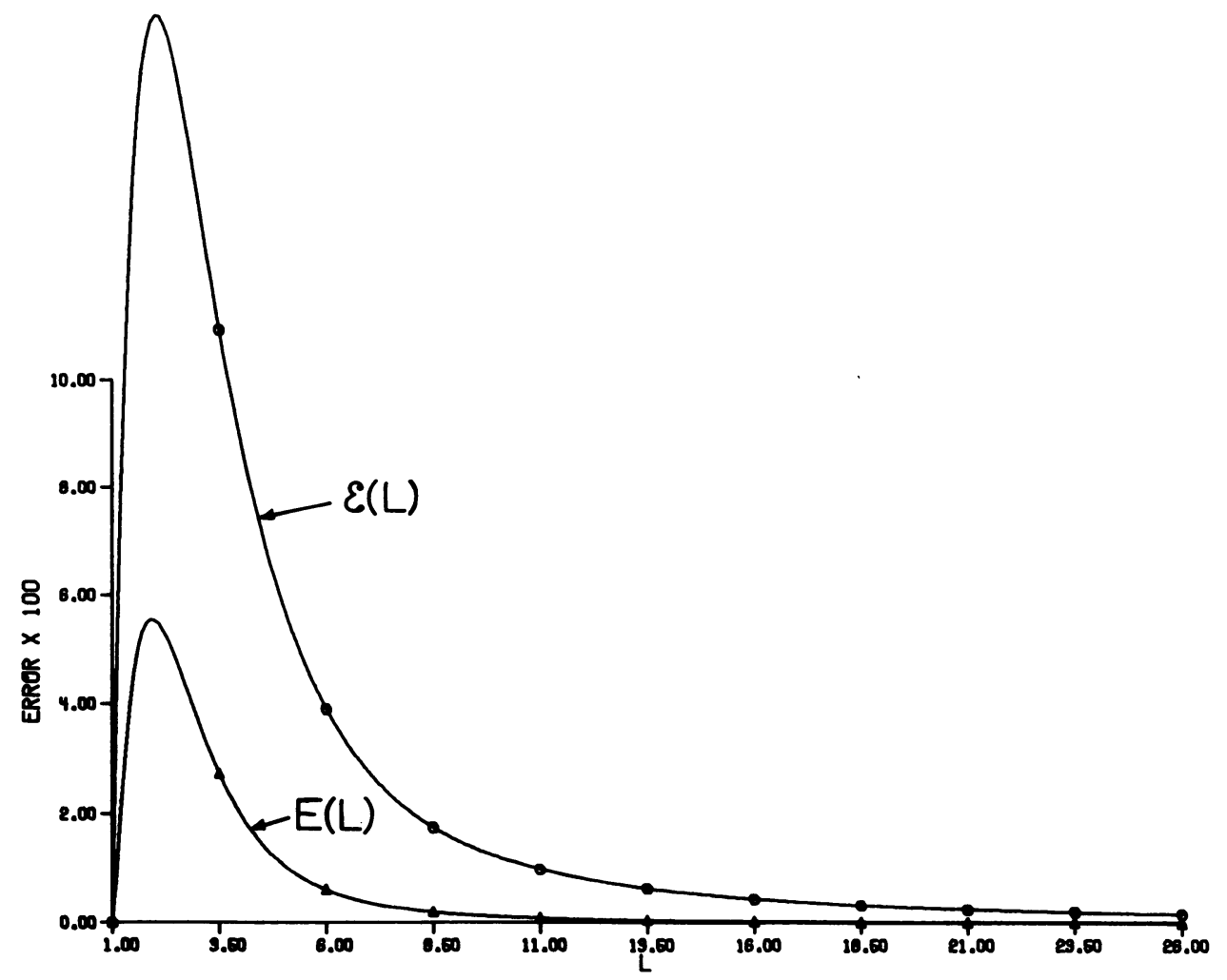

FIgURE 2. Error Criteria, First Example 
interval lengths. The interval length is indicated by the intersection of the curve with the $x$ axis. Inspection of the curves reveals that for interval lengths greater than about 6 , very good results are obtained over most of the domain. In particular, after the interval length exceeded 6 , the maximum value of $y$ changed by less than .2 per cent. In Fig. 2, the two error criteria for this example $E(L)$ and $E(L)$ are shown as functions of the interval length. Both criteria move rapidly toward zero.

The same problem was solved using the alternate superposition suggested by Eq. (29). These results are shown in Fig. 3. Note that better convergence near the end of the intervals was obtained. It is also interesting to note that in this case "convergence" was from above.

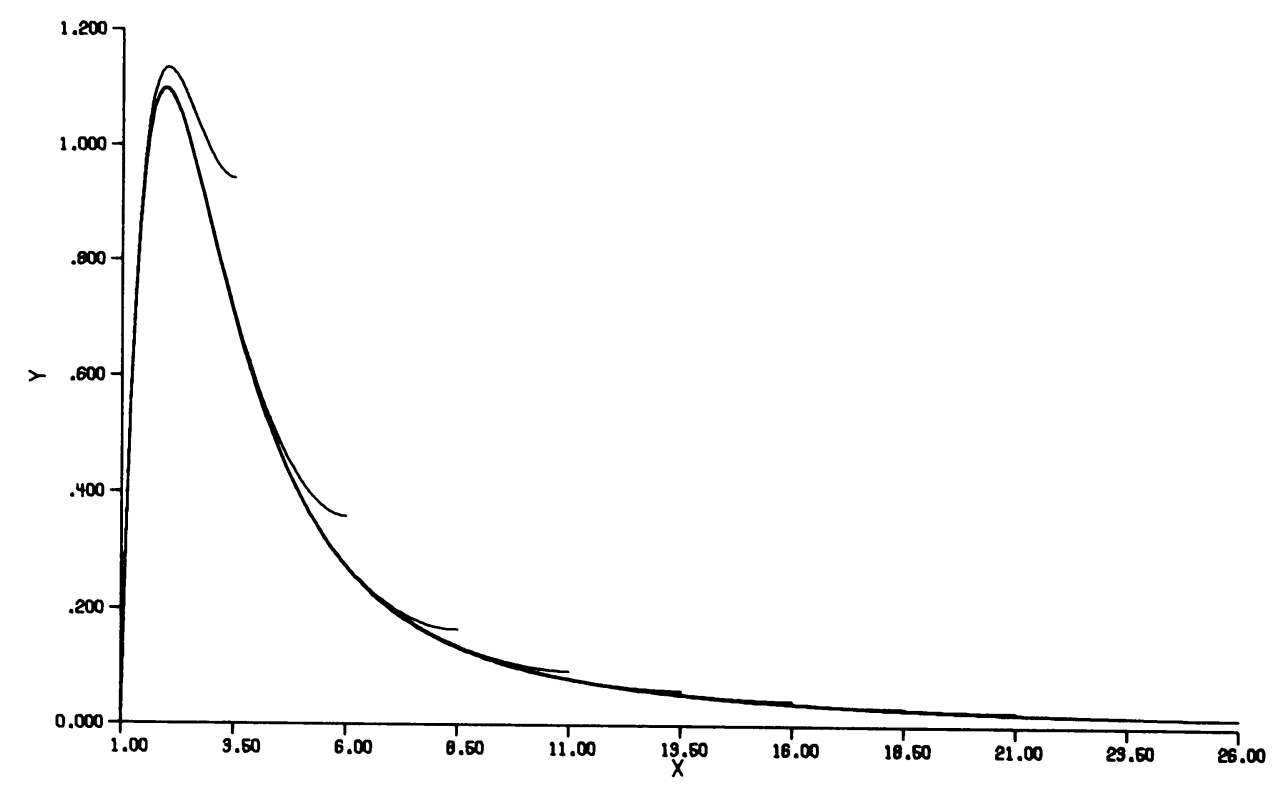

FIGURE 3. Alternate Invariant Imbedding Solution, First Example

As a second example, the following problem was solved using the invariant imbedding method.

$$
\begin{gathered}
\frac{d^{2} y}{d x^{2}}-.01\left(1+5 \frac{\sin x}{x}\right) y=-6.5 \sin (2 x) e^{-x} \\
y(0)=-.5, \quad \lim _{x \rightarrow \infty} y(x)=0 .
\end{gathered}
$$

Note that the coefficient of $y$ in Eq. (31) changes sign twice in the domain $0<x \leqslant \infty$. The results are shown in Fig. 4. The error criterion $E(L)$ is presented in Fig. 5. Note that good results are obtained for values of $L$ greater than about 24 . 


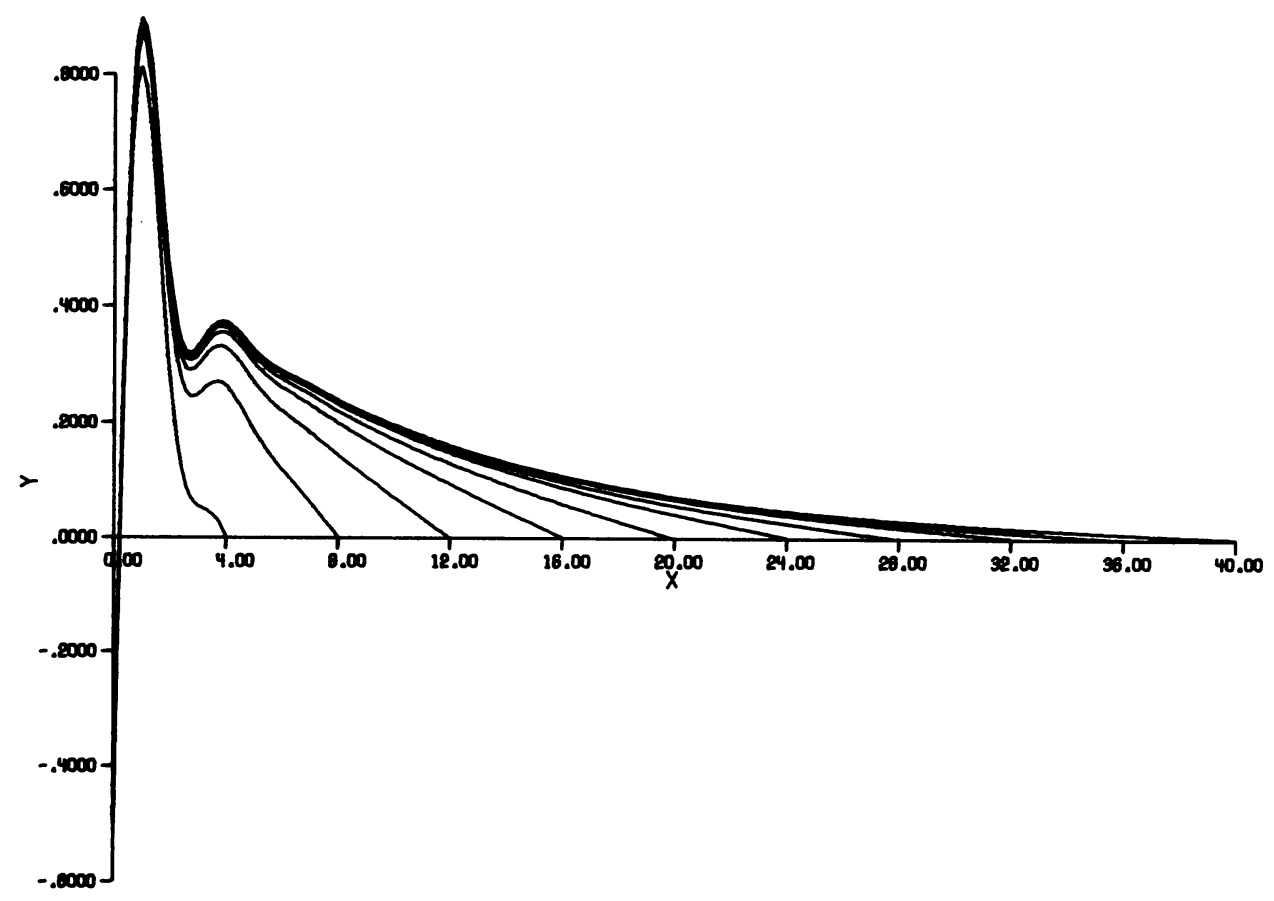

FIGURE 4. Invariant Imbedding Solution, Second Example

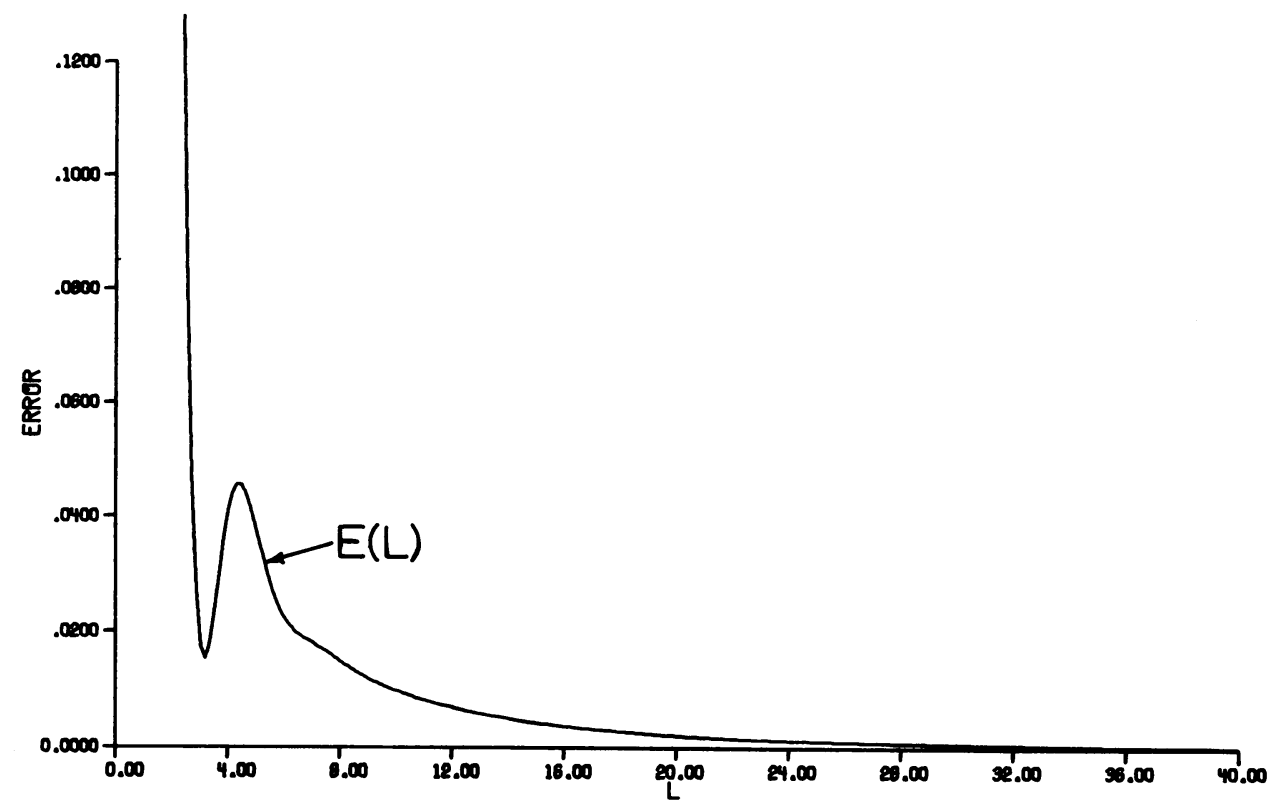

FIGURE 5. Error Criterion, Second Example 
6. Discussion. It has been demonstrated that invariant imbedding can be used to good advantage in the solution of linear two-point boundary value problems defined on a semi-infinite domain. The Riccati equations obtained by the invariant imbedding technique have proven to be numerically stable. Attempts to use the usual methods of solution of the TPBVP often fail in certain ill-conditioned problems (e.g., widely separated roots of the characteristic equation). Past experience has shown that the numerical stability of the invariant imbedding formulation permits the easy solution of such problems.

The invariant imbedding method offers several criteria for the determination of an appropriate interval length. Since the limiting values of $E(L)$ and $E(L)$ can be obtained, a priori error criteria can be used in the algorithm. In the event that additional accuracy is required, it is a simple task to save the appropriate initial conditions required for restart of the program. As a result of limited investigations, it would appear that a reasonable stopping condition would be based on reducing $E(L)$ to the order of $10^{-4}$.

Output can be obtained for as many or as few points as desired and at any location in the domain of the problem. Of course, three differential equations are required for each point.

School of Aeronautics and Astronautics Purdue University

West Lafayette, Indiana 47907

1. T. N. ROBERTSON, "The linear two-point boundary value problem on an infinite interval," Math. Comp., v. 25, 1971, pp. 475-481. MR 46 \#2878.

2. D. W. ALSPAUGH, H. H. KAGIWADA \& R. KALABA, "Dynamic programming, invariant imbedding and thin beam theory," Internat. J. Engrg. Sci., v. 7, 1969, pp. 1117-1126. MR 40 \#2267.

3. D. W. AlSPAUGH, H. H. KAGIWADA \& R. KALABA, "Application of invariant imbedding to the buckling of columns," J. Computational Phys., v. 5, 1970, pp. 56-69. MR 40 \#177.

4. D. W. ALSPAUGH \& R. KALABA, Direct Derivation of Invariant Imbedding Equations for Beams from a Variational Principle, RAND Corp., RM-5995-PR, March 1969.

5. P. B. BAILEY \& G. M. WING, "Some recent developments in invariant imbedding with applications," J. Mathematical Phys., v. 6, 1965, pp. 453-462. MR 30 \#2882.

6. J. CASTI \& R. KALABA, Imbedding Methods in Applied Mathematics, Addison-Wesley, Reading, Mass., 1973.

7. G. H. MEYER, Initial Value Methods for Boundary Value Problems, Academic Press, New York, 1974. 